\title{
Interval Fuzzy Linear Programming Models to Solve Interval-valued Fuzzy Zero-Sum Games
}

\author{
Stephanie Loi Brião ${ }^{1}$ Graçaliz Pereira Dimuro ${ }^{2}$ Catia Maria dos Santos Machado ${ }^{1}$ \\ ${ }^{1}$ Programa de Pós-Graduação em Modelagem Computacional \\ ${ }^{2}$ Programa de Pós-Graduação em Computação, Universidade Federal do Rio Grande, Rio Grande, Brazil
}

\begin{abstract}
In Game theory, there are situations in which it is very difficult to characterize the private information of each player. In this case, the payoffs can be given by approximate values, represented by fuzzy numbers. Whenever there is uncertainty in the modeling of those fuzzy numbers, interval fuzzy numbers may be used. This paper introduces two approaches for the solution of interval-valued fuzzy zero-sum games. First, we extend the CamposVerdegay model, which uses triangular fuzzy numbers for the modeling of uncertain payoffs, to consider interval-valued fuzzy payoffs. Then, defining a ranking method for interval fuzzy numbers that induces a total order, we generalize the intervalvalued Campos-Verdegay model to consider payoffs modeled as any type of interval fuzzy numbers. In both models, we establish an Interval Fuzzy Linear Programming problem for each player, which are reduced to classical Linear Programming problems, used in the solution of classical zero-sum games. We show that the solutions are of the same nature of the parameters defining the game, corresponding to an uncertain predicate of type: "the value of the game is in the interval $\vartheta "$.
\end{abstract}

Keywords: interval fuzzy numbers, ranking interval fuzzy numbers, interval fuzzy zero-sum games, interval fuzzy linear programming

\section{Introduction}

Game theory [1] is a well known important basis to simulate several situations where multiple players interact strategically for decision making and support [2]. In many applications, the players only know their own characteristics, and must make decisions while having to estimate the characteristics of the other participants of the interaction, which configures a game of incomplete information [3]. When probabilities are assigned for the different types of the interacting agents, we have a game of imperfect information [4]. However, there are cases in which it is very difficult to characterize the private information of each player (e.g., ability, level of effort), in order to establish the probabilities of the types that they may assume. In these situations, the payoffs are given by approximate (not probabilistic) values.

Fuzzy set theory [5] is an excellent basis for studying the kind of game in which the payoffs are represented by fuzzy numbers. In the literature, there is a large amount of research on fuzzy games, mainly focused on matrix fuzzy games and noncooperative fuzzy games, the majority on zero-sum games, which are strictly competitive games.[6]

Campos and Verdegay $[7,8]$ introduced a twoperson zero-sum game with payoffs given by triangular fuzzy numbers, whose solution is based on the establishment of a Fuzzy Linear Programming (FLP) problem for each player, which are reduced to Linear Programming (LP) models by adopting ranking methods for triangular fuzzy numbers. Amaral and Gomide $[9,10]$ analyzed equilibrium strategies and developed computational methods for the resolution of fuzzy zero-sum games, based on evolutionary computation, in $\alpha$-levels and decomposition algorithms. See also the works by Dutta and Gupta [11] and Maeda [12].

Whenever there is uncertainty in the modeling of the fuzzy numbers (e.g., when they are given by a group of different domain experts), interval fuzzy numbers are frequently used, in order to represent a range of fuzzy numbers, varying from the most pessimistic (most uncertain) evaluation to most the optimistic (less uncertain). Observe that intervalvalued fuzzy sets allows to deal not only with vagueness (lack of sharp class boundaries), but also with uncertainty (lack of information) [13, 14, 15, 16].

This paper introduces two approaches for twoperson interval-based fuzzy zero-sum games, where interval-valued fuzzy payoffs are considered. First, the Campos-Verdegay model, which uses triangular fuzzy numbers for the modeling of uncertain payoffs, is extended to consider interval fuzzy payoffs. Then, defining a ranking method for interval fuzzy numbers that induces a total order, we generalize the interval-valued Campos-Verdegay model to consider payoffs modeled by any type of interval fuzzy number, so enlarging the scope of the applications and allowing for further extensions. In both models, we establish an Interval Fuzzy Linear Programming (IFLP) problem for each player, which are reduced to classical LP problems, used in the solution of classical zero-sum games. We show that the solutions are of the same nature of the parameters of the game, corresponding to an uncertain predicate of type: "the value of the game is in the interval $\vartheta$ ".

The paper is organized as follows. Section 2 
presents preliminary concepts, introducing a ranking method for interval fuzzy numbers. Basic concepts on zero-sum games are presented in Sect. 3. In Sect. 4, we discuss fuzzy zero-sum games, presenting the original Campos-Verdegay model and generalizing it considering Ezzati et al.'s ranking method. Section 5 introduces interval-valued fuzzy zero-sum games: the interval-valued Campos-Verdegay model and a model based on the total order introduced in Sect. 2. Section 6 is the Conclusion.

\section{Preliminary Concepts}

A fuzzy set is defined by means of a graded membership function. Given an universe $\mathbb{U}$ and a fuzzy subset $A$ of $\mathbb{U}$, the membership function $\varphi_{A}: \mathbb{U} \rightarrow[0,1]$ gives the grade $\varphi_{A}(x)$ with which an element $x \in \mathbb{U}$ belongs to $A$. A fuzzy subset $A$ of $\mathbb{U}$ can be then represented by a set of ordered pairs, given by $A=\left\{\left(x, \varphi_{A}(x)\right) \mid x \in \mathbb{U}\right\}$. The support of $F$ is defined as the set

$$
\operatorname{supp}_{A}=\left\{x \in \mathbb{U} \mid \varphi_{A}(x)>0\right\} .
$$

The core of $A$ is defined as the set core $_{A}=\{x \in$ $\left.\mathbb{U} \mid \varphi_{A}(x)=1\right\}$. For $0 \leq \alpha \leq 1$, the $\alpha$-cuts of $A$ are defined as the classic subsets of $\mathbb{U}$ given by

$$
A[\alpha]= \begin{cases}\left\{x \in \mathbb{U} \mid \varphi_{A}(x) \geq \alpha\right\} & \text { if } 0<\alpha \leq 1 \\ \widehat{\operatorname{supp}_{A}} & \text { if } \alpha=0\end{cases}
$$

where $\operatorname{supp}_{A}$ is the support of $A$ defined in Eq. (1) and $\widehat{\operatorname{supp}_{A}}$ is the closure of $\operatorname{supp}_{A}$. A fuzzy set is completely defined by its $\alpha$-cuts. [17]

A fuzzy set $\tilde{N}$ is called a fuzzy number whenever $\varphi_{\tilde{N}}$ is defined on the set of real numbers $\mathbb{R}$, and the following conditions hold: (i) all $\alpha$-cuts of $\tilde{N}$ are non empty closed intervals in $\mathbb{R}$ and (ii) the support of $\tilde{N}$ is bounded. [5, 18]

There are several kinds of fuzzy numbers, according to their membership functions. A trapezoidal fuzzy number $\tilde{N}$ is given by a tuple $\tilde{N}=$ $\left(\eta_{1}, \eta_{2}, \sigma, \beta\right)$, where core $_{\tilde{N}}=\left[\eta_{1}, \eta_{2}\right], \sigma$ is its left fuzziness related to $\eta_{1}, \beta$ is its right fuzziness related to $\eta_{2}$ [19], and its membership function is given by:

$$
\varphi_{\tilde{N}}(x)= \begin{cases}\frac{x-\eta_{1}+\sigma}{\sigma} & \text { if } \eta_{1}-\sigma \leq x \leq \eta_{1} \\ 1 & \text { if } \eta_{1} \leq x \leq \eta_{2} \\ \frac{\eta_{2}+\beta-x}{\beta} & \eta_{2} \leq x \leq \eta_{2}+\beta \\ 0 & \text { otherwise. }\end{cases}
$$

Whenever $\eta_{1}=\eta_{2}=\eta$, then $\tilde{N}$ is a triangular fuzzy number denoted by $\tilde{N}=(\eta, \sigma, \beta)$.

Considering its membership function, a fuzzy number $\tilde{N}$ can be represented in a parametric form as $\tilde{N}=(\underline{\tilde{N}}(t), \overline{\tilde{N}}(t))$, for $0 \leq t \leq 1$ [20]. For example, given Eq. (3), a trapezoidal fuzzy number $\tilde{N}$ can be represented in a parametric form as:

$$
\tilde{N}=(\underline{\tilde{N}}(t), \overline{\tilde{N}}(t))=\left(\eta_{1}-\sigma+\sigma t, \eta_{2}+\beta-\beta t\right),
$$

for $0 \leq t \leq 1$. The parametric form of a triangular fuzzy number is similarly defined.

The magnitude of a fuzzy number in a parametric form is given by: [20]

$$
\operatorname{Mag}(\tilde{N})=\frac{1}{2} \int_{0}^{1}(\underline{\tilde{N}}(t)+\overline{\tilde{N}}(t)+\underline{\tilde{N}}(1)+\overline{\tilde{N}}(1)) F(t) \mathrm{d} t
$$

where the function $F$ is is a non-negative and increasing function defined on $[0,1]$ with $F(0)=0$, $F(1)=1$ and $\int_{0}^{1} F(t) \mathrm{d} t=\frac{1}{2} . F$ is considered as a weighting function. In actual applications, the function $F$ can be chosen according to the actual situation. In this paper, we use $F(t)=t$. The magnitude of fuzzy numbers may be used for ranking fuzzy numbers [20]. Ezzati et al. [19] introduced another complementary magnitude of a fuzzy number $\tilde{N}=(\underline{\tilde{N}}(t), \overline{\tilde{N}}(t))$, given by:

$$
\operatorname{Mag}^{\prime}(\tilde{N})=\frac{1}{2} \int_{0}^{1}\left(\underline{\tilde{N}^{\prime}}(t)-\overline{\tilde{N}^{\prime}}(t)+\overline{\tilde{N}}(1)-\underline{\tilde{N}}(1)\right) \mathrm{d} t .
$$

In order to rank two fuzzy numbers $\tilde{N}$ and $\tilde{M}$, Ezzati et al. [19] defined a ranking value $R$ :

$$
\begin{aligned}
R(\tilde{N}, \delta) & =\operatorname{Mag}(\tilde{N})+\delta \operatorname{Mag}^{\prime}(\tilde{N}) \\
R(\tilde{M}, \delta) & =\operatorname{Mag}(\tilde{M})+\delta \operatorname{Mag}^{\prime}(\tilde{M}), \\
\text { where } \delta & = \begin{cases}0 & \text { if } \operatorname{Mag}(\tilde{N}) \neq \operatorname{Mag}(\tilde{M}) \\
1 & \text { if } \operatorname{Mag}(\tilde{N})=\operatorname{Mag}(\tilde{M}),\end{cases}
\end{aligned}
$$

with $M a g$ and $M a g^{\prime}$ defined in Eqs. (5) and (6), respectively. The ranking of $\tilde{N}$ and $\tilde{M}$ is given by: [19]

$$
\tilde{N} \preceq \tilde{M} \Leftrightarrow R(\tilde{N}, \delta) \leq R(\tilde{M}, \delta) .
$$

There are several ranking methods for fuzzy numbers (see, e.g., [21, 22, 23, 24, 25]). We adopted the one proposed by Ezzati et al. [19] because it induces a total order, and can be easily combined with the interval order by Costa et al. [26] in order to obtain a ranking method for interval fuzzy numbers, which also induces a total order.

Consider the set of real intervals $\mathbb{I R}$ and let $U=\{[a, b] \in \mathbb{I R} \mid 0 \leq a \leq b \leq 1\}$ be the set of subintervals of the unit interval $[0,1]$. An interval fuzzy subset $\mathcal{A}$ of a universe $\mathbb{U}$ is defined as the set of ordered pairs $\mathcal{A}=\left\{\left(x, \nu_{\mathcal{A}}(x)\right) \mid x \in \mathbb{U}\right\}$, where $\nu_{A}: \mathbb{U} \rightarrow U$ is the interval-valued membership function of $\mathcal{A}$, which provides the interval membership grade $\nu_{\mathcal{A}}(x)$ containing the uncertain membership grade with which an element $x \in \mathbb{U}$ belongs to $\mathcal{A}$. Whenever the interval membership function $\nu_{\mathcal{A}}$ is continuous ${ }^{1}$, then there exist continuous functions $\varphi_{\mathcal{A}^{l}}, \varphi_{\mathcal{A}^{u}}: \mathbb{U} \rightarrow[0,1]$, called, respectively, the lower membership function (LMF) and the upper membership function (UMF), such that, for every $x \in \mathbb{U}$, it holds that $\nu_{\mathcal{A}}(x)=\left[\varphi_{\mathcal{A}^{l}}(x), \varphi_{\mathcal{A}^{u}}(x)\right]$, where $\varphi_{\mathcal{A}^{l}}(x) \leq \varphi_{\mathcal{A}^{u}}(x)$. [18]

The inner and outer supports of an interval fuzzy set $\mathcal{A}$ are defined, respectively, by $\operatorname{lsupp}_{\mathcal{A}}=\{x \in$ $\left.\mathbb{U} \mid \varphi_{\mathcal{A}^{l}}(x)>0\right\}$ and $\operatorname{usupp}_{\mathcal{A}}=\left\{x \in \mathbb{U} \mid \varphi_{\mathcal{A}^{u}}(x)>\right.$ $0\}$, and its core is $\operatorname{core}_{\mathcal{A}}=\left\{x \in \mathbb{U} \mid \nu_{\mathcal{A}}(x)=\{x \in\right.$ $\left.\mathbb{U} \mid \varphi_{\mathcal{A}^{l}}(x)=\varphi_{\mathcal{A}^{u}}(x)=1\right\}$. For $\left[\alpha_{1}, \alpha_{2}\right] \in U$, the $\left[\alpha_{1}, \alpha_{2}\right]$-cuts of $\mathcal{A}$ are defined by: [18]

\footnotetext{
${ }^{1}$ The continuity of interval functions was defined by Moore as an extension of the continuity of real functions. More information on this subject can be seen in $[27,28]$.
} 
$\mathcal{A}\left[\alpha_{1}, \alpha_{2}\right]=$

$\begin{cases}\left\{x \in \mathbb{U} \mid \nu_{\mathcal{A}}(x) \geq_{\mathbb{I R}}\left[\alpha_{1}, \alpha_{2}\right]\right\}, & \text { if } \alpha_{1} \neq 0 \\ \mathcal{A}^{l}[0] \cap\left\{x \in \mathbb{U} \mid \nu_{\mathcal{A}}(x) \geq_{\mathbb{I R}}\left[\alpha_{1}, \alpha_{2}\right]\right\}, & \text { if } \alpha_{1}=0 \wedge \alpha_{2} \neq 0 \\ \mathcal{A}^{l}[0] \cap \mathcal{A}^{u}[0], & \text { if } \alpha_{1}=\alpha_{2}=0,\end{cases}$

where $\geq_{\mathbb{I R}}$ is an interval order relation ${ }^{2}, \mathcal{A}^{l}[0]$ and $\mathcal{A}^{u}[0]$ are the closures of the supports $\operatorname{lsupp}_{\mathcal{A}}$ and $\operatorname{usupp}_{\mathcal{A}}$, respectively. As in the classical fuzzy theory, an interval fuzzy set is completely determined by its $\left[\alpha_{1}, \alpha_{2}\right]$-cuts. [18]

An interval fuzzy number $\widehat{N}$ is defined as an interval fuzzy set of $\mathbb{R}$ satisfying the properties: (1) the $\left[\alpha_{1}, \alpha_{2}\right]$-cuts and the core of $\widehat{N}$ are real intervals of IIR; (2) lsupp $_{\widehat{N}}$ and $\operatorname{ssupp}_{\widehat{N}}$ are bounded [18]. A trapezoidal interval fuzzy number may be represented as an ordered pair of fuzzy numbers $\widehat{N}=$ $\left(\tilde{N}^{l}, \tilde{N}^{u}\right)$, where $\tilde{N}^{l}$ and $\tilde{N}^{u}$ are called the lower and upper generator fuzzy numbers of $\widehat{N}$, respectively, specified by the respective LMF and UMF.

An interval fuzzy number $\widehat{N}$ is called trapezoidal whenever both LMF and UMF are given as in Eq. (3), defining the lower and upper trapezoidal fuzzy numbers $\tilde{N}^{l}=\left(\eta_{1}^{l}, \eta_{2}^{l}, \sigma^{l}, \beta^{l}\right)$ and $\tilde{N}^{u}=$ $\left(\eta_{1}^{u}, \eta_{2}^{u}, \sigma^{u}, \beta^{u}\right)$, respectively. Similarly, we define a triangular interval fuzzy number.

Now we generalize the ranking method introduced by Ezzati et al. [19] for interval fuzzy numbers. Consider two trapezoidal interval fuzzy numbers $\widehat{N}=\left(\tilde{N}^{l}, \tilde{N}^{u}\right)$ and $\widehat{M}=\left(\tilde{M}^{l}, \tilde{M}^{u}\right)$, where the lower and upper generator fuzzy numbers $\tilde{N}^{l}, \tilde{N}^{u}$, $\tilde{M}^{l}$ and $\tilde{M}^{u}$ are defined in the parametric forms, and the respective magnitudes are defined as in Equations (5) and (6). Then, the lower and upper ranking values of $\widehat{N}$ and $\widehat{M}$ are given as:

$$
\begin{aligned}
R\left(\tilde{N}^{l}, \delta^{l}\right) & =\operatorname{Mag}\left(\tilde{N}^{l}\right)+\delta^{l} \operatorname{Mag}^{\prime}\left(\tilde{N}^{l}\right) \\
R\left(\tilde{M}^{l}, \delta^{l}\right) & =\operatorname{Mag}\left(\tilde{M}^{l}\right)+\delta^{l} \operatorname{Mag}^{\prime}\left(\tilde{M}^{l}\right) \\
R\left(\tilde{N}^{u}, \delta^{u}\right) & =\operatorname{Mag}\left(\tilde{N}^{u}\right)+\delta^{u} \operatorname{Mag}^{\prime}\left(\tilde{N}^{u}\right) \\
R\left(\tilde{M}^{u}, \delta^{u}\right) & =\operatorname{Mag}\left(\tilde{M}^{u}\right)+\delta^{u} \operatorname{Mag}^{\prime}\left(\tilde{M}^{u}\right),
\end{aligned}
$$

where $\delta^{l}$ and $\delta^{u}$ are given by Eq. (9). Define:

$$
\begin{aligned}
& \widehat{N} \preceq \widehat{M} \Leftrightarrow \\
& {\left[R\left(\tilde{N}^{l}, \delta^{l}\right), R\left(\tilde{N}^{u}, \delta^{u}\right)\right] \leq_{\mathbb{I R}}^{C}\left[R\left(\tilde{M}^{l}, \delta^{l}\right), R\left(\tilde{M}^{u}, \delta^{u}\right)\right],}
\end{aligned}
$$

where $\leq_{\mathbb{I R}}^{C}$ is the Costa et al.'s total order in the set of real intervals $\mathbb{I R}[26]$. It is immediate that

Proposition $1 \preceq$ is a total order relation in the set of trapezoidal fuzzy numbers.

Example 1 Let $\widehat{A}=\left(\tilde{A}^{l}, \tilde{A}^{u}\right)$ be a trapezoidal interval fuzzy number, defined by the generator fuzzy numbers $\tilde{A}^{l}=(-1,1,2,2)$ and $\tilde{A}^{u}=(-1,1,3,3)$, and let $\widehat{B}=\left(\tilde{B}^{l}, \tilde{B}^{u}\right)$ be a triangular interval fuzzy number, defined by the generator fuzzy numbers

\footnotetext{
${ }^{2}$ E.g., for $X=\left[x_{1}, x_{2}\right], Y=\left[y_{1}, y_{2}\right] \in \mathbb{I R}$, the KulishMiranker order relation [28] is defined by $X \leq_{\mathrm{IR}}^{K M} Y \Leftrightarrow$ $x_{1} \leq y_{1} \wedge x_{2} \leq y_{2}$ and the Costa total order relation [26] is given by $X \leq \leq_{\mathbb{I R}}^{C} Y \Leftrightarrow x_{2}<y_{2} \vee\left(x_{2}=y_{2} \wedge y_{1} \leq x_{1}\right)$.
}

$\tilde{B}^{l}=(0,1,1)$ and $\tilde{B}^{u}=(0,2,2)$, as shown in Fig. 1 . The parametric forms of the generator fuzzy numbers of $\widehat{A}$ and $\widehat{B}$, for $0 \leq t \leq 1$, are:

$$
\begin{aligned}
\tilde{A}^{l} & =\left(\underline{\tilde{A}^{l}}(t), \overline{\tilde{A}^{l}}(t)\right)=(-3+2 t, 3-2 t) \\
\tilde{A}^{u} & =\left(\underline{\tilde{A}^{u}}(t), \overline{\tilde{A}^{u}}(t)\right)=(-4+3 t, 4-3 t) \\
\tilde{B}^{l} & =\left(\underline{\tilde{B}^{l}}(t), \overline{\tilde{B}^{l}}(t)\right)=(-1+t, 1-t) \\
\tilde{B}^{u} & =\left(\underline{\tilde{B}}^{u}(t), \overline{\tilde{B}^{u}}(t)\right)=(-2+2 t, 2-2 t) .
\end{aligned}
$$

The magnitudes are $\operatorname{Mag}\left(\tilde{A}^{l}\right)=\operatorname{Mag}\left(\tilde{A}^{u}\right)=0$, $\operatorname{Mag}\left(\tilde{B}^{l}\right)=\operatorname{Mag}\left(\tilde{B}^{u}\right)=0, \quad \operatorname{Mag}^{\prime}\left(\tilde{A}^{l}\right)=\frac{1}{2} \int_{0}^{1} 6 \mathrm{~d} t=3$, $\operatorname{Mag}^{\prime}\left(\tilde{A}^{u}\right)=\frac{1}{2} \int_{0}^{1} 8 \mathrm{~d} t=4, \quad \operatorname{Mag}^{\prime}\left(\tilde{B}^{l}\right)=\frac{1}{2} \int_{0}^{1} 2 \mathrm{~d} t=1$, $\operatorname{Mag}^{\prime}\left(\tilde{B}^{u}\right)=\frac{1}{2} \int_{0}^{1} 4 \mathrm{~d} t=2 . \quad$ Since $\delta^{l}=\delta^{u}=1$, then one has that $\left[R^{l}\left(\tilde{A}^{l}, \delta^{l}\right), R^{u}\left(\tilde{A}^{u}, \delta^{u}\right)\right]=[3,4]$, $\left[R^{l}\left(\tilde{B}^{l}, \delta^{l}\right), R^{u}\left(\tilde{B}^{u}, \delta^{u}\right)\right]=[1,2]$, and, thus, $\widehat{B} \prec \widehat{A}$.

\section{Two-person Zero-Sum Games}

A zero-sum game is a non-cooperative game in which a player's gain (or loss) of utility is exactly balanced by the losses (or gains) of the utility of the other player(s). If the total gains of the playerss are added up, and the total losses are subtracted, they will sum to zero. A zero-sum game is also called a strictly competitive game. Zero-sum games are most often solved with the minimax theorem, which is closely related to linear programming duality (as we show below), or with Nash equilibrium. [29]

Usually, one represents a zero-sum game by the matrix of gains (payoffs, rewards), according to the chosen strategy in the interaction. The players adopt mixed strategies, that is, probabilistic strategies. The solution method for zero-sum two-person games is based on the establishment of a LP problem for each player. [29]

Let $I=\{1, \ldots, m\}$ and $J=\{1, \ldots, n\}$ be the set of possible actions to be chosen by the players I and II, respectively. The payoff matrix $\mathcal{P}$ of the rewards of the player $\mathrm{I}$ is given by $\mathcal{P}=\left[p_{i j}\right]_{i \in I, J \in J}$. In a zero-sum game, the payoff matrix $\mathcal{Q}$ of the rewards of the player II is given by $\mathcal{Q}=-\mathcal{P}$.

Consider the mixed strategies of the players I and II, given by the following probabilistic vectors:

$$
\begin{gathered}
X=\left\{x=\left(x_{1}, \cdots, x_{m}\right) \in \mathbb{R}^{m} \mid\right. \\
\left.\sum_{i=1}^{m} x_{i}=1, x_{i} \geq 0, i=1, \cdots, m\right\} \\
Y=\left\{y=\left(y_{1}, \cdots, y_{n}\right) \in \mathbb{R}^{n} \mid\right. \\
\left.\sum_{j=1}^{n} y_{j}=1, y_{j} \geq 0, j=1, \cdots, n\right\} .
\end{gathered}
$$

The expected reward is given by: [30]

$$
\vartheta(x, y)=\sum_{i=1}^{m} \sum_{j=1}^{n} x_{i} p_{i j} y_{j}=x \mathcal{P} y
$$

Since the objetives of each player are just the opposite, each player should obtain the following values:

$$
v_{\mathrm{I}}=\max _{x \in X} \min _{y \in Y} x \mathcal{P} y \text { and } v_{\mathrm{II}}=\min _{y \in Y} \max _{x \in X} x \mathcal{P} y \text {. }
$$




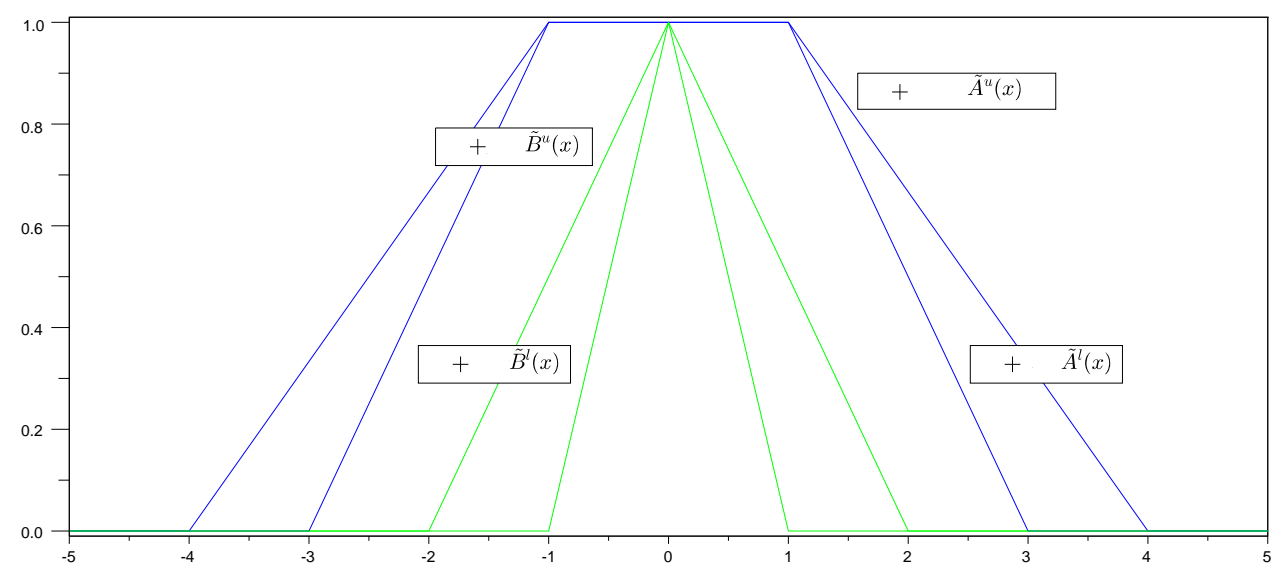

Figure 1: Interval fuzzy numbers $\widehat{A}=\left(\tilde{A}^{l}, \tilde{A}^{u}\right)$ and $\widehat{B}=\left(\tilde{B}^{l}, \tilde{B}^{u}\right)$

The equilibrium solution is the pair of strategies $(x, y)$ that satisfies the condition: $v_{\mathrm{I}}=v_{\mathrm{II}} \cdot[30]$

Considering $\left.\mathcal{P}\right|_{j}=\left[p_{1 j}, \ldots, p_{m j}\right]^{T}$, one has that $v_{\mathrm{I}}=\max _{x \in X} \min _{y \in Y}(x \mathcal{P} y)=\max _{x \in X} \min _{j \in J}\left(\left.x \mathcal{P}\right|_{j}\right)=\max _{x \in X} \min _{j \in J} \sum_{i=1}^{m} p_{i j} x_{i}$.

So, define the following linear programming model:

$$
\begin{gathered}
\max _{x \in X} \min _{j \in J} \sum_{i=1}^{m} p_{i j} x_{i} \\
\text { s.t. }: x_{1}+x_{2}+\ldots+x_{m}=1 \\
x_{i} \geq 0, i=1, \ldots, m .
\end{gathered}
$$

For the sake of simplicity, consider an auxiliary variable $z$, such that: $[9,10,7]$

$\max z$

$$
\begin{array}{ll}
\text { s.t. } & : p_{1 j} x_{1}+\cdots+p_{m j} x_{m} \geq z, j=1, \ldots, n \\
& x_{1}+x_{2}+\ldots+x_{m}=1 \\
& x_{i} \geq 0, i=1, \ldots, m .
\end{array}
$$

Since $\max z$ is equivalent to $\min 1 / z=s_{1}+\ldots+$ $s_{m}$, where $s$ is an auxiliary variable, we obtain the following linear programming:

$$
\begin{aligned}
& \min s_{1}+\cdots+s_{m} \\
& \text { s.t. }: p_{1 j} s_{1}+\cdots+p_{m j} s_{m} \geq 1, j \in J \\
& \quad s_{i} \geq 0, i \in I
\end{aligned}
$$

The procedure to obtain $v_{\text {II }}$ is analogous, considering an auxiliary variable $w$ in order to simplify the linear programming analogous to (17), and the fact that $\min w$ is equivalent to $\max 1 / w=r_{1}+\ldots+r_{n}$, where $r$ is an auxiliary variable. Then, we have the following linear programming:

$$
\begin{aligned}
\max r_{1} & +\cdots+r_{n} \\
\text { s.t. } & : p_{i 1} r_{1}+\cdots+p_{i n} r_{n} \leq 1, i \in I \\
& r_{j} \geq 0, j \in J
\end{aligned}
$$

Finally, considering that $s_{i}=\frac{x_{i}}{z}$ and $r_{j}=\frac{y_{j}}{w}$, with $i \in I$ and $j \in J$, one obtains the solution $x^{*}=s \times z$ and $y^{*}=r \times w$. Then the final value of the game is given by $\vartheta=x^{*} \mathcal{P} y^{*}$.

\section{Fuzzy two-person Zero-Sum Games}

Whenever one considers the uncertainty/vagueness of the parameters in actual applications, it is possible to work with fuzzy zero-sum games. The solution method is based on the establishment of a Fuzzy Linear Programming (FLP) problem for each player, as generalizations of that conventionally used in the solution of classical games (Sect. 3, see also $[7,9,10,31,32])$. To solve a FLP problem, auxiliary models resulting from the application of a method for ranking fuzzy numbers are considered, transforming the FLP into a classical crisp LP.

In fuzzy zero-sum games, the payoff matrix of the rewards of the player $I$ is given by the matrix of fuzzy numbers $\tilde{\mathcal{P}}=\left[\tilde{p}_{i j}\right]_{i \in I, j \in J}$. By a construction analogous to presented in Sect. 3, considering the fuzzy payoff matrix $\tilde{\mathcal{P}}$ and the fuzzy independent term $\tilde{b}$, the solution is obtained by the following FLP models, for the players I and II, respectively:

$$
\begin{aligned}
& \min s_{1}+\cdots+s_{m} \\
& \text { s.t. : } \tilde{p}_{1 j} s_{1}+\cdots+\tilde{p}_{m j} s_{m} \succeq \tilde{b}_{j}, j \in J \\
& s_{i} \succeq 0, i \in I \\
& \max r_{1}+\cdots+r_{n} \\
& \text { s.t. : } \tilde{p}_{i 1} r_{1}+\cdots+\tilde{p}_{i n} r_{n} \preceq \tilde{b}_{i}, i \in I \\
& r_{j} \succeq 0, j \in J .
\end{aligned}
$$

where $\preceq(\succeq)$ is a fuzzy order relation.

\subsection{The Campos-Verdegay Model}

Campos and Verdegay $[7,8]$ proposed a solution method for the above FLP, where the fuzzy constraint set is replaced by a convex set using the following relations [7, page 277, Eq. (2)], which was 
first proposed by Delgado et al. [33]:

$$
\tilde{p}_{j} s \geq \tilde{b}_{j}-\tilde{t}_{j}(1-\alpha) \text { and } \tilde{p}_{i} r \leq \tilde{b}_{i}+\tilde{q}_{i}(1-\alpha),
$$

with $i \in I, j \in J, \alpha \in] 0,1]$, where $\tilde{t}$ and $\tilde{q}$ are fuzzy parameters expressing the maximal violations that the players permit in the accomplishment of the constraints, and $\leq(\geq)$ is any order relation between triangular fuzzy numbers that preserves the ranking if the fuzzy numbers are multiplied by a positive scalar. Some specific fuzzy order relations transforming the FLP into crisp LP are used, e.g., using the following order relations [7, page 279, Eq. (d)]:

$$
p_{j} s \geq b_{j}-d_{j}(1-\alpha) \text { and } p_{i} r \leq b_{i}+e_{i}(1-\alpha) \text {, }
$$

with $i \in I, j \in J, \alpha \in] 0,1], d_{j}=\operatorname{core}_{\tilde{t}_{j}}, e_{j}=\operatorname{core}_{\tilde{q}_{i}}$ and $b=$ core $_{\tilde{b}}$, one obtains the crisp LP:

$$
\begin{aligned}
& \min s_{1}+\cdots+s_{m} \\
& \text { s.t. : } p_{1 j} s_{1}+\cdots+p_{m j} s_{m} \geq b_{j}-d_{j}(1-\alpha) \text {, } \\
& j \in J, \alpha \in] 0,1] \\
& s_{i} \geq 0, i \in I \\
& \max r_{1}+\cdots+r_{n} \\
& \text { s.t. : } p_{i 1} r_{1}+\cdots+p_{i n} r_{n} \leq b_{i}+e_{j}(1-\alpha) \text {, } \\
& i \in I, \alpha \in] 0,1] \\
& r_{j} \leq 0, j \in J
\end{aligned}
$$

Observe that, since a FLP model is transformed into classical LPs, then, the solution is analogous to the construction presented in Sect. 3.

\subsection{The Proposed Model Based on Ezzati et al.'s Ranking Method}

Observe that sometimes it is not possible to find a solution by Campos-Verdegay model, since it was established for triangular fuzzy numbers only. Then, we introduce a variation of the CamposVerdegay method, called generalized CamposVerdegay method, in order to consider the ranking method proposed by Ezzati et al. [19] (see Sect. 2), which derives a total order and can be easily applied to any kind of fuzzy numbers. ${ }^{3}$

Consider the fuzzt payoff matrix $\widetilde{\mathcal{P}}$, and define the matrix of ranking values of the payoffs by:

$$
R(\tilde{\mathcal{P}}, \delta)=\left[R\left(\tilde{p}_{i j}, \delta\right)\right]_{i \in I, j \in J},
$$

where

$$
R\left(\tilde{p}_{i j}, \delta\right)=\operatorname{Mag}\left(\tilde{p}_{i j}\right)+\delta M a g^{\prime}\left(\tilde{p}_{i j}\right),
$$

with $M a g$ and $M a g^{\prime}$ given, respectively, by Eq. (5) and Eq. (6), and

$$
\delta= \begin{cases}0 & \text { if } \forall i, i^{\prime} \in I \forall j, j^{\prime} \in J: \\ & i \neq i^{\prime} \vee j \neq j^{\prime} \Rightarrow \operatorname{Mag}\left(\tilde{p}_{i j}\right) \neq \operatorname{Mag}\left(\tilde{p}_{i^{\prime} j^{\prime}}\right) \\ 1 & \text { otherwise. }\end{cases}
$$

Consider the FLP model given in (21) and (22), and the relations (23) adapted as follows:

$$
\begin{aligned}
R\left(\tilde{p}_{j}, \delta\right) s & \geq R\left(\tilde{b}_{j}, \delta\right)-R\left(\tilde{t}_{j}, \delta\right)(1-\alpha) \\
R\left(\tilde{p}_{i}, \delta\right) r & \leq R\left(\tilde{b}_{i}, \delta\right)+R\left(\tilde{q}_{i}, \delta\right)(1-\alpha),
\end{aligned}
$$

\footnotetext{
${ }^{3}$ An initial proposal of this method was discussed in [32].
}

with $i \in I, j \in J, \alpha \in] 0,1]$, where $\tilde{t}$ and $\tilde{q}$ are fuzzy parameters expressing the maximal violations that the players permit in the accomplishment of the constraints. Then one obtains the following LP model for the players I and II, respectively:

$$
\begin{aligned}
& \min s_{1}+\cdots+s_{m} \\
& \text { s.t. }: \sum_{i=1}^{m} R\left(\tilde{p}_{i j}, \delta\right) s_{i} \geq R\left(\tilde{b}_{j}, \delta\right)-R\left(\tilde{t}_{j}, \delta\right)(1-\alpha), \\
& \left.\left.\quad s_{i} \geq 0, i \in I, \alpha \in\right] 0,1\right] \\
& \max r_{1}+\cdots+r_{n} \\
& \text { s.t. : } \sum_{j=1}^{n} R\left(\tilde{p}_{i j}, \delta\right) r_{j} \leq R\left(\tilde{b}_{i}, \delta\right)+R\left(\tilde{q}_{i}, \delta\right)(1-\alpha), \\
& \quad r_{j} \geq 0, j \in J .
\end{aligned}
$$

Since a FLP model is transformed into classical LPs, the solution is analogous to which we presented in Sect. 3. Considering the solutions $x^{*}$ and $y^{*}$, the value of the game is obtained as $x^{*} R(\tilde{\mathcal{P}}, \delta) y^{*}$.

\section{Interval-valued Fuzzy Zero-Sum Games}

Whenever one considers uncertainty/vagueness in both the parameters and the membership functions modelling those parameters, it is possible to work with interval fuzzy zero-sum games. The solution method for two-person interval fuzzy zerosum games is based on the establishment of an IFLP problem for each player, as generalizations of what we present for the the solution of fuzzy games (Sect. 4). To solve an IFLP problem, we first split the model obtaining two FLPs (namely, the Lower an the Upper FLPs), and then we proceed as explained in Sect. 4, using a method for ranking fuzzy numbers, transforming each FLP into a classical LP.

The payoffs of an interval fuzzy zero-sum game are given by interval fuzzy numbers $\hat{p}_{i j}=\left(\tilde{p}_{i j}^{l}, \tilde{p}_{i j}^{u}\right)$. We consider an interval fuzzy payoff matrix $\tilde{\mathcal{P}}$ of the rewards of the player I, defined by the interval fuzzy matrix $\hat{\mathcal{P}}=\left(\tilde{\mathcal{P}}^{l}, \tilde{\mathcal{P}}^{u}\right)$, represented by the pair composed by the lower and upper payoff matrices, given, respectively, by: $\tilde{\mathcal{P}}^{l}=\left[\tilde{p}_{i j}^{l}\right]_{i \in I, j \in J}$ and $\tilde{\mathcal{P}}^{u}=$ $\left[\tilde{p}_{i j}^{u}\right]_{i \in I, j \in J}$. Then, according to (21) and (22), one obtains the lower FLP:

$$
\begin{aligned}
\min s_{1}+\cdots+s_{m} & \\
\text { s.t. : } & \tilde{p}_{1 j}^{l} s_{1}+\cdots+\tilde{p}_{m j}^{l} s_{m} \succeq \tilde{b}_{j}^{l}, j \in J \\
& s_{i} \succeq 0, i \in I \\
\max r_{1}+\cdots+r_{n} & \\
\text { s.t. : } & \tilde{p}_{i 1}^{l} r_{1}+\cdots+\tilde{p}_{i n}^{l} r_{n} \preceq \tilde{b}_{i}^{l}, i \in I \\
& r_{j} \succeq 0, j \in J .
\end{aligned}
$$

where $\preceq(\succeq)$ is a fuzzy order relation. The upper FLP is analogous, considering the upper payoff matrix $\tilde{\mathcal{P}}^{u}$. In the following subsections, we introduce two approaches for solving (34)-(35). 


\subsection{Interval-valued Campos-Verdegay Model}

In this section, we extend the Campos-Verdegay method (Sect. 4) to an interval fuzzy approach. Considering that the payoffs are given by triangular interval fuzzy numbers, and taking the lower FLPs given in (34)-(35), and interval fuzzy versions of the relations (23) and (24), we obtain:

$$
\begin{gathered}
\min s_{1}+\cdots+s_{m} \\
\text { s.t. : } p_{1 j}^{l} s_{1}+\cdots+p_{m j}^{l} s_{m} \underset{j \in J, \alpha \in] 0,1]}{\geq} b_{j}^{l}-d_{j}^{l}(1-\alpha), \\
j \in J \\
s_{i} \geq 0, i \in I \\
\max r_{1}+\cdots+r_{n} \\
\text { s.t. : } p_{i 1}^{l} r_{1}+\cdots+p_{i n}^{l} r_{n} \leq \begin{array}{l}
\left(36+e_{j}^{l}(1-\alpha),\right. \\
i \in I, \alpha \in] 0,1]
\end{array} \\
r_{j} \leq 0, j \in J .
\end{gathered}
$$

The upper FLP is obtained analogously, considering the upper payoff matrix $\tilde{\mathcal{P}}^{u}$.

Since we get LP models, the solution is analogous to what was presented in Sect. 3. It follows that the solutions are obtained as $x^{l^{*}}=s^{l} \times z^{l}, y^{l^{*}}=$ $r^{l} \times w^{l}, x^{u *}=s^{u} \times z^{u}$ and $y^{u *}=r^{u} \times w^{u}$, that is, $x^{*}=\left(s^{l} \times z^{l}, s^{u} \times z^{u}\right)$ and $y^{*}=\left(r^{l} \times w^{l}, r^{u} \times w^{u}\right)$, where $s_{i}=\frac{x_{i}}{z}$ and $r_{j}=\frac{y_{i}}{w}$, for auxiliary variables $z$ and $w$, as defined in Sect. 3 , and $i \in I, j \in J$.

The final interval value of the game is specified by $\vartheta^{l}=x^{l^{*}} \mathcal{P}^{l} y^{l^{*}}$ and $\vartheta^{u}=x^{u *} \mathcal{P}^{u} y^{u *}$, so that:

$$
\vartheta= \begin{cases}{\left[\vartheta^{l}, \vartheta^{u}\right]} & \text { if } \vartheta^{l} \leq \vartheta^{u} \\ {\left[\vartheta^{u}, \vartheta^{l}\right]} & \text { if } \vartheta^{l}>\vartheta^{u} .\end{cases}
$$

\subsection{The Proposed Model Based on a Total Order}

In this section, we introduce a model based on the total interval fuzzy order relation defined in Eq. (12), called generalized Intervalvalued Campos-Verdegay Model. Let $R\left(\tilde{\mathcal{P}}^{l}, \delta^{l}\right)$ and $R\left(\tilde{\mathcal{P}}^{u}, \delta^{u}\right)$ be the matrices of the ranking values of the lower and upper payoffs, respectively, defined as in Eq. (27). Considering the lower FLP given in (34)-(35), and the relations (30) and (31) defined for lower payoffs, we obtain:

$$
\begin{aligned}
& \min s_{1}+\cdots+s_{m} \\
& \begin{array}{c}
\text { s.t. : } \sum_{i=1}^{m} R\left(\tilde{p}_{i j}^{l}, \delta^{l}\right) s_{i} \geq R\left(\tilde{b}_{j}^{l}, \delta^{l}\right)-R\left(\tilde{t}_{j}^{l}, \delta^{l}\right)(1-\alpha), \\
j \in J, \alpha \in] 0,1]
\end{array} \\
& s_{i} \geq 0, i \in I \\
& \max r_{1}+\cdots+r_{n} \\
& \begin{array}{c}
\text { s.t. : } \sum_{j=1}^{n} R\left(\tilde{p}_{i j}^{l}, \delta^{l}\right) r_{j} \leq R\left(\tilde{b}_{i}^{l}, \delta^{l}\right)+R\left(\tilde{q}_{i}^{l}, \delta^{l}\right)(1-\alpha), \\
i \in I, \alpha \in] 0,1]
\end{array} \\
& r_{j} \geq 0, j \in J,
\end{aligned}
$$

where $\delta^{l}$ is defined as in Eq. (29). The upper FLP is constructed analogously, considering the upper payoff matrix $\tilde{\mathcal{P}}^{u}$, the relations (30) and (31) defined for upper payoffs and $\delta^{u}$ as defined in Eq. (29).
Since we now have LP models, the solution is analogous to what was presented in Sect. 3. Consider the optimal solutions $x^{*}=\left(s^{l} \times z^{l}, s^{u} \times z^{u}\right)$ and $y^{*}=\left(r^{l} \times w^{l}, r^{u} \times w^{u}\right)$, where $s_{i}=\frac{x_{i}}{z}$ and $r_{j}=\frac{y_{i}}{w}$, for auxiliary variables $z$ and $w$, as defined in Sect. 3, and $i \in I, j \in J$. The final interval value of the game is given as in Eq. (38), where $\vartheta^{l}=x^{l^{*}} R\left(\mathcal{P}^{l}, \delta^{l}\right) y^{l^{*}}$ and $\vartheta^{u}=x^{u *} R\left(\mathcal{P}^{u}, \delta^{u}\right) y^{u *}$.

\subsection{Example}

Consider an interval fuzzy zero-sum game whose interval fuzzy payoff matrix $\widehat{\mathcal{P}}=\left(\tilde{\mathcal{P}}^{l}, \tilde{\mathcal{P}}^{u}\right)$ is specified by the lower and upper fuzzy payoff matrices:

$$
\begin{aligned}
\tilde{\mathcal{P}}^{l} & =\left[\begin{array}{ccc}
(180,0.5,0) & (156,0.5,2) & (90,0.5,0.5) \\
(90,1,0.5) & (180,2,0.5) & (155,4,4) \\
(180,0.5,0.5) & (156,1,1) & (177,2,2)
\end{array}\right] \\
\tilde{\mathcal{P}}^{u} & =\left[\begin{array}{ccc}
(180,2,0) & (156,2,5) & (90,2,2) \\
(90,4,2) & (180,4,2) & (155,6,6) \\
(180,2,2) & (156,3,3) & (177,4,4)
\end{array}\right] .
\end{aligned}
$$

Let the independent term $\hat{b}=\left(\tilde{b}^{l}, \tilde{b}^{u}\right)$ be given by $\tilde{b}^{l}=\tilde{b}^{u}=(1,0,0)$, and the interval fuzzy parameters $\hat{t}=\left(\tilde{t}^{l}, \tilde{t}^{u}\right)$ and $\hat{q}=\left(\tilde{q}^{l}, \tilde{q}^{u}\right)$, expressing the maximal violations that the players permit in the accomplishment of the constraints, be defined by $\tilde{t}^{l}=(0.10,0.01,0.005), \tilde{t}^{u}=(0.10,0.03,0.02)$, $\tilde{q}^{l}=(0.15,0.005,0.01)$ and $\tilde{q}^{u}=(0.10,0.02,0.03)$.

Since we are adopting triangular interval fuzzy numbers, then it is possible to apply the proposed Interval-valued Campos-Verdegay Model, introduced in Sect. 5.1. According (36)-(37), the following LP for both lower and upper fuzzy payoff matrices is obtained:

$$
\begin{aligned}
& \min s_{1}+s_{2}+s_{3} \\
& \text { s.t. : } 180 s_{1}+90 s_{2}+180 s_{3} \geq 1-0.1(1-\alpha) \\
& 156 s_{1}+180 s_{2}+156 s_{3} \geq 1-0.1(1-\alpha) \\
& 90 s_{1}+155 s_{2}+177 s_{3} \geq 1-0.1(1-\alpha) \\
&\left.\left.s_{1}, s_{2}, s_{3} \geq 0, \alpha \in\right] 0,1\right] \\
& \max r_{1}+r_{2}+r_{3} \\
& \text { s.t. : } 180 r_{1}+156 r_{2}+90 r_{3} \leq 1+0.15(1-\alpha) \\
& 90 r_{1}+180 r_{2}+155 r_{3} \leq 1+0.15(1-\alpha) \\
& 180 r_{1}+156 r_{2}+177 r_{3} \leq 1+0.15(1-\alpha) \\
&\left.\left.r_{1}, r_{2}, r_{3} \geq 0, \alpha \in\right] 0,1\right] .
\end{aligned}
$$

The solution is obtained as

$$
\begin{aligned}
x^{l^{*}}=x^{u^{*}} & =(0.1301,0.2104,0.6594) \\
y^{l^{*}}=y^{u^{*}} & =(0.2104,0.7893,0.0000) \\
z(\alpha) & \left.\left.=\frac{161.0026}{1-0.1(1-\alpha)}, \alpha \in\right] 0,1\right] \\
w(\alpha) & \left.\left.=\frac{161.0026}{1+0.15(1-\alpha)}, \alpha \in\right] 0,1\right]
\end{aligned}
$$

and the interval fuzzy value of the interval fuzzy game game is given by $\vartheta^{l}=\vartheta^{u}=161.0026$, that is, it is around 161.0026.

On the other hand, applying the proposed generalized Interval-valued Campos-Verdegay method, introduced in Sect. 5.2, the matrix of magnitudes of 
the interval fuzzy payoff matrix $\widehat{\mathcal{P}}$, using Eq. (5), is:

$$
\begin{aligned}
\operatorname{Mag}\left(\tilde{\mathcal{P}}^{l}\right) & =\left[\begin{array}{ccc}
179.95 & 156.12 & 90 \\
89.95 & 179.87 & 155 \\
180 & 156 & 177
\end{array}\right] \\
\operatorname{Mag}\left(\tilde{\mathcal{P}}^{u}\right) & =\left[\begin{array}{ccc}
179.83 & 156 & 90 \\
89.83 & 179.83 & 155 \\
180 & 156 & 177
\end{array}\right]
\end{aligned}
$$

Since, by Eq. (29), $\delta^{l}=\delta^{u}=0$, then, by Eq. (28), it holds that $R\left(\tilde{\mathcal{P}}^{l}, \delta^{l}\right)=\operatorname{Mag}\left(\tilde{\mathcal{P}}^{l}\right)$ and $R\left(\tilde{\mathcal{P}}^{u}, \delta^{u}\right)=$ $\operatorname{Mag}\left(\tilde{\mathcal{P}}^{u}\right)$. Analogously, the magnitudes of the independent term $\hat{b}$ and of the interval fuzzy violation parameters $\hat{t}$ and $\hat{q}$ are given by $\operatorname{Mag}\left(\tilde{b}^{l}\right)=$ $\operatorname{Mag}\left(\tilde{b}^{u}\right)=1, \operatorname{Mag}\left(\tilde{t}^{l}\right)=0.0995, \operatorname{Mag}\left(\tilde{t}^{u}\right)=$ 0.0991, $\operatorname{Mag}\left(\tilde{q}^{l}\right)=0.1504$ and $\operatorname{Mag}\left(\tilde{q}^{u}\right)=0.1508$, and the ranking values are similarly obtained. As in (39)-(40), considering the lower fuzzy payoff matrix $\tilde{\mathcal{P}}^{l}$, one has the following LP:

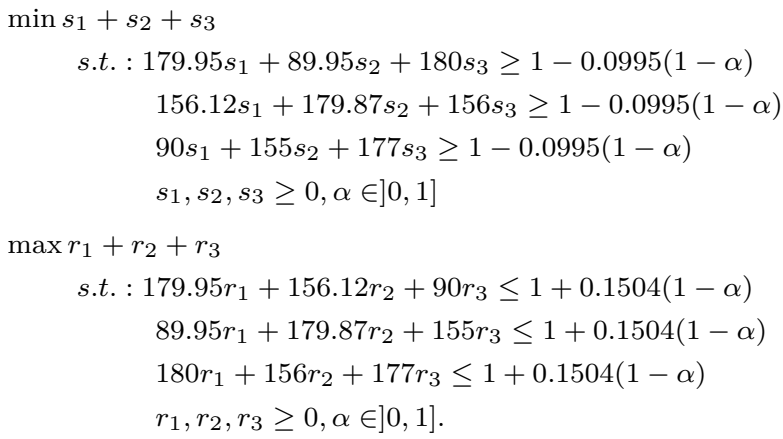

And, considering the upper fuzzy payoff matrix $\tilde{\mathcal{P}}^{u}$, one has the following LP:

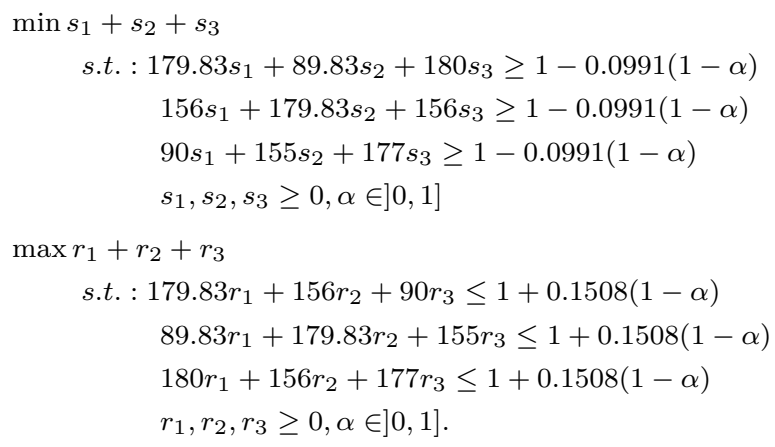

The solution is obtained as

$$
\begin{aligned}
x^{l^{*}} & =(0.1301,0.2104,0.6593) \\
x^{u^{*}} & =(0.0000,0.2105,0.7894) \\
y^{l^{*}} & =(0.2091,0.7898,0.0009) \\
y^{u^{*}} & =(0.2090,0.7916,0.0000) \\
z^{l}(\alpha) & \left.\left.=\frac{160.9700}{1-0.0995(1-\alpha)}, \alpha \in\right] 0,1\right] \\
z^{u}(\alpha) & \left.\left.=\frac{161.0900}{1-0.0991(1-\alpha)}, \alpha \in\right] 0,1\right] \\
w^{l}(\alpha) & \left.\left.=\frac{160.9700}{1+0.1504(1-\alpha)}, \alpha \in\right] 0,1\right] \\
w^{u}(\alpha) & \left.\left.=\frac{161.0900}{1+0.1508(1-\alpha)}, \alpha \in\right] 0,1\right]
\end{aligned}
$$

and the interval fuzzy value of the interval fuzzy game game is given by $\vartheta^{l}=160.9700$ and $\vartheta^{u}=161.0900$, that is, it is in the interval [160.9700, 161.0900].

The results showed that the solution provided by the Interval-valued Campos-Verdegay method is encapsulated by the solution obtained with the generalized Interval-valued Campos-Verdegay method, showing the lower and upper bounds for the approximate solution. We have used triangular interval fuzzy numbers in order to be able to compare the solutions obtained by both methods. Observe that in the generalized Interval-valued Campos-Verdegay model it is possible to adopt also trapezoidal fuzzy numbers, which is not true for the Interval-valued Campos-Verdegay model.

\section{Conclusion}

This paper introduced two approaches for twoperson interval-based fuzzy zero-sum games, where the payoffs are modeled as interval fuzzy numbers, in order to be applied in strategic interactions where two kinds of uncertainty may be considered: the uncertainty in the characteristics of each player (consequently, the vagueness of the payoffs of the game) and the lack of information for the establishment of the membership functions of the fuzzy numbers.

The first approach consisted in an extension of the Campos-Verdegay model, which uses triangular fuzzy numbers for the modeling of uncertain payoffs, in order to consider interval-valued fuzzy payoffs. For the second approach, we defined a ranking method for interval fuzzy numbers that induces a total order, based on Ezzati et al.'s and Costa et al.'s methods. Then, we generalized the intervalvalued Campos-Verdegay model to consider payoffs modeled as any type of interval fuzzy numbers. In both models, we establish an IFLP problem for each player, which are reduced to classical LP problems, used in the solution of classical zero-sum games. We show that the solutions are of the same nature of the parameters defining the game, corresponding to an uncertain predicate of type: "the value of the game is in the interval $\vartheta "$.

Future work will be concerned with the development of applications in decision making.

\section{Acknowledgments}

This work was supported by CAPES and CNPq (Proc. No. 481283/2013-7, 306970/2013-9).

\section{References}

[1] K. Leyton-Brown and Y. Shoham. Essentials of game theory: A concise, multidisciplinary introduction. Morgan \& Claypool, http://www.morganclaypool.com/, 2008.

[2] J. A. Reneke. A game theory formulation of decision making under conditions of uncertainty and risk. Nonlinear Analysis: Theory, Methods \& Applications, 71(12):e1239 - e1246, 2009.

[3] C. Harsanyi. Games with incomplete information. The American Economic Review, 85:291303, 1999. 
[4] C. Wang, W. Tang, and R. Zhao. Static Bayesian games with finite fuzzy types and the existence of equilibrium. Information Sciences, 178(24):4688 - 4698, 2008.

[5] L. A. Zadeh. Fuzzy sets. Information and Control, 8(3):338-353, 1965.

[6] J.-F. Dang and I-H. Hong. The Cournot game under a fuzzy decision environment. Computers and Mathematics with Applications, 59(9):3099-3109, 2010.

[7] L. Campos. Fuzzy linear programming models to solve fuzzy matrix games. Fuzzy Sets and Systems, 32:275-289, 1989.

[8] L. Campos and J. L. Verdegay. Linear programming problems and ranking of fuzzy numbers. Fuzzy Sets and Syst., 32(1):1 - 11, 1989.

[9] W. Amaral and F. Gomide. An algorithm to solve two-person non-zero sum fuzzy games. In O. Castillo et al., eds., Theoretical Advances and Applications of Fuzzy Logic and Soft Computing, Advances in Soft Computing, pages 296-302. Springer, Berlin, 2007.

[10] W. Amaral and F. Gomide. A coevolutionary approach to solve fuzzy games. In R. Bello et al., eds., Granular Computing: At the Junction of Rough Sets and Fuzzy Sets, pages 121 - 130. Springer, Berlin, 2008.

[11] B. Dutta and S. K. Gupta. On nash equilibrium strategy of two-person zero-sum games with trapezoidal fuzzy payoffs. Fuzzy Information and Engineering, 6(3):299 - 314, 2014.

[12] T. Maeda. On characterization of equilibrium strategy of two-person zero-sum games with fuzzy payoffs. Fuzzy Sets and Systems, 139(2):283 - 296, 2003.

[13] B. C. Bedregal, G. P. Dimuro, R. H. N. Santiago, and R. H. S. Reiser. On interval fuzzy S-implications. Information Sciences, 180(8):1373 - 1389, 2010.

[14] G. P. Dimuro, B. C. Bedregal, R. H. N. Santiago, and R. H. S. Reiser. Interval additive generators of interval t-norms and interval tconorms. Information Sciences, 181(18):3898 - 3916, 2011.

[15] D. Dubois and H. Prade. Interval-valued fuzzy sets, possibility theory and imprecise probability. In Proc. Joint 4th Conf. of the European Society for Fuzzy Logic and Technology and the 11th Rencontres Francophones sur la Logique Floue et ses Ap., pages 314-319, Barcelona, 2005. Universidad Polytecnica de Catalunya.

[16] W. A. Lodwick. Preface. Reliable Computing, 10(4):247-248, 2004.

[17] G. J. Klir and B. Yuan. Fuzzy Sets and Fuzzy Logics: Theory and Applications. Prentice Hall, Upper Saddle River, 1995.

[18] G. P. Dimuro. On interval fuzzy numbers. In Workshop-School on Theoretical Computer Science, pages 3-8, Los Alamitos, 2011. IEEE.

[19] R. Ezzati, T. Allahviranloo, S. Khezerloo, and
M. Kheserloo. An approach for ranking of fuzzy numbers. Expert Systems with Applications, 39:690 - 695, 2012.

[20] S. Abbasbandy and T. Hajjari. A new approach for ranking of trapezoidal fuzzy numbers. Computers and Mathematics with Applications, 57:413 - 419, 2009.

[21] T. C. Asmus and G. P. Dimuro. On fuzzy probabilities in Bayesian games. In 2011 WorkshopSchool on Theoretical Computer Science, pages 25-31, Los Alamitos, 2011. IEEE.

[22] T. C. Asmus, B. R. C. Bedregal, and G. P. Dimuro. On interval fuzzy Bayesian games: Preliminary results. In Decision Making and Soft Computing, Proc. of the 11th Intl. FLINS Conference, chapter 24, pages 131-136. World Scientific, Singapore, 2014.

[23] T. C. Asmus and G. P. Dimuro. A total order for symmetric triangular (interval) fuzzy numbers. Mathware \& Soft Computing Magazine, 20(1):76-114, 2013.

[24] H. Deng. Comparing and ranking fuzzy numbers using ideal solutions. Applied Mathematical Modelling, 38(5-6):1638 - 1646, 2014.

[25] M. Brunelli and J. Mezei. How different are ranking methods for fuzzy numbers? a numerical study. International Journal of Approximate Reasoning, 54(5):627 - 639, 2013.

[26] C. G. Costa, B. Bedregal, and A. D. Dória Neto. Atanassov's intuitionistic fuzzy probability and Markov chains. Knowledge-Based Systems, 43:52 - 62, 2013.

[27] G. P. Dimuro, A. C. R. Costa, , and D. M. Claudio. A coherence space of rational intervals for a construction of IR. Reliable Computing, 6(2):139-178, 2000.

[28] R. E. Moore, R. B. Kearfott, and M. J. Cloud. Introduction to Interval Analysis. SIAM, Philadelphia, 2009.

[29] M. Larbani. Non cooperative fuzzy games in normal form: A survey. Fuzzy Sets and Systems, 160(22):3184 - 3210, 2009.

[30] I. Nishizaki and M. Sakawa. Fuzzy and Multiobjective Games for Conflict Resolution. Physica-Verlag, Heidelberg, 2001.

[31] J. J. Buckley and L. Jowers. Fuzzy two-person zero-sum games. In Monte Carlo Methods in Fuzzy Optimization, volume 222 of Studies in Fuzziness and Soft Computing, pages 165-173. Springer, Berlin, 2008.

[32] S. L. Briao, G. P. Dimuro, and C. M. S. Machado. Linear programming modeling for solving fuzzy zero-sum games. In 2013 2nd Workshop-School on Theoretical Computer Science, pages 84-91, Los Alamitos, 2013. IEEE.

[33] M. Delgado, J.L. Verdegay, and M.A. Vila. A general model for fuzzy linear programming. Fuzzy Sets and Systems, 29(1):21 - 29, 1989. 